\title{
Observation of Superconductivity in Highly Cr-Doped GaP Single Crystals
}

\author{
J.B. $\operatorname{Gosk}^{a, b}$, G. Strzelecka ${ }^{c}$, G. Kowalski ${ }^{a}$, A. Hruban ${ }^{c}$, \\ M. KAMińskA ${ }^{a}$, A. TwARdowski ${ }^{a}$ AND J. GronkOWskI ${ }^{a}$ \\ ${ }^{a}$ Institute of Experimental Physics, Warsaw University \\ Hoża 69, 00-681 Warsaw, Poland \\ ${ }^{b}$ Faculty of Physics, Warsaw University of Technology \\ Koszykowa 75, 00-662 Warsaw, Poland \\ ${ }^{c}$ Institute of Electronic Materials Technology \\ Wólczyńska 133, 01-919 Warsaw, Poland
}

\begin{abstract}
Studying bulk GaP, highly doped with $\mathrm{Cr}$, and searching for possible ferromagnetic semiconductor in view of spintronic applications, we found superconducting behavior of this material unexpectedly. Magnetization techniques and X-ray diffraction were applied to study these crystals. Magnetization revealed superconducting features up to about $6 \mathrm{~K}$ and X-ray studies showed that superconductivity might be related to small size $\mathrm{Cr}$ precipitates.
\end{abstract}

PACS numbers: 74.10.+v, 75.50.Pp, 61.72.Vv, 61.72.Dd, 81.10.Dn

\section{Introduction}

Superconductivity was first observed for mercury in 1911 [1]. Since then it has been found in many metallic elements, alloys and doped semiconductors, and critical temperature for these materials extends from $0.001 \mathrm{~K}$ for $\mathrm{Rh}$ up to $138 \mathrm{~K}$ for record-holder $\left(\mathrm{Hg}_{0.8} \mathrm{Tl}_{0.2}\right) \mathrm{Ba}_{2} \mathrm{Ca}_{2} \mathrm{Cu}_{3} \mathrm{O}_{8.33}$ [2]. Studies performed in recent years have shown that there are several ways to cause non-superconducting elements to superconduct, including application of pressure, irradiation, quenched growth on a cold substrate, epitaxial templating in the form of thin film, or chemical doping [3].

In this paper we report on results of magnetic properties and X-ray diffraction (XRD) measurements performed on highly Cr-doped GaP single crystals. Studying highly Cr-doped GaP we found superconductivity of this material at temperatures below about $6 \mathrm{~K}$.

Bulk Cr metal is not a superconductor. It is antiferromagnetic element with the Néel temperature $T_{\mathrm{N}}=312 \mathrm{~K}$. However, there have been reports about su- 
perconducting chromium at temperatures up to $1.52 \mathrm{~K}$ in ion beam sputtered bcc Cr thin films on soft glass [4]. The critical temperature $T_{\mathrm{c}}$ has been found to depend on the ion species used for film deposition. Also, superconducting chromium in metastable fcc phase, fabricated by epitaxially sandwiching $\mathrm{Cr}$ between gold layers was observed [5-8]. The authors were searching for correlation of $T_{\mathrm{c}}$ value and layer thickness. They found the highest $T_{\mathrm{c}}$, equal to $3 \mathrm{~K}$, for thin epitaxial metal film sandwiches of $\mathrm{Au} / \mathrm{Cr} / \mathrm{Au}$ where the $\mathrm{Au}$ (100) layer thicknesses were approximately $5 \mathrm{~nm}$ and $\mathrm{Cr}$ layer was $2 \mathrm{~nm}$ thick. However, superconducting properties of the $\mathrm{Cr}$ layer changed with time and $T_{\mathrm{c}}$ dropped from $3 \mathrm{~K}$ to $2.16 \mathrm{~K}$ for samples kept at room temperature after only one day. This was probably due to interdiffusion.

Theoretical calculations made for the electronic structure of fcc Cr placed superconducting transition temperature around $2.5 \mathrm{~K}$, which is smaller than the highest temperature of $10 \mathrm{~K}$ derived from the measurements for $\mathrm{Au} / \mathrm{Cr} / \mathrm{Au}$ sandwiches [9]. Recent review on superconducting elements [3] quotes $T_{\mathrm{c}}$ equal to $3 \mathrm{~K}$ for $\mathrm{Cr}$.

Superconductivity has been also found for granular contacts to two-dimensional electron gases employing $\mathrm{Sn}: \mathrm{Cr}: \mathrm{Au}$ multilayers with critical temperatures of $5-7.5 \mathrm{~K}[10]$.

\section{Samples}

Bulk GaP crystals of cubic structure, intentionally doped with Cr were grown by liquid encapsulated Czochralski (LEC) method. Metallic chromium was added to the melt and incorporated to the crystal during growth process. In four growth processes performed, the initial chromium concentration in the melt changed in the range of $1.25 \times 10^{20} \mathrm{~cm}^{-3}$ to $6 \times 10^{20} \mathrm{~cm}^{-3}$. Chromium concentration in some of the crystals was measured using glow discharge mass spectrometry (GDMS) technique and chromium segregation coefficient was obtained as about 0.001. GDMS measurements also indicated presence of unintentional sulphur and silicon dopants with concentration up to half of that of $\mathrm{Cr}$. Other impurities were on the level below $10^{15} \mathrm{~cm}^{-3}$. Since $\mathrm{S}$ and $\mathrm{Si}$ are shallow donors in $\mathrm{GaP}$, it was expected that $\mathrm{Cr}$ configuration should be $\mathrm{Cr}^{3+}\left(d^{3}\right) / \mathrm{Cr}^{2+}\left(d^{4}\right)$ with energy level close to the midgap, $1.2 \mathrm{eV}$ below $\mathrm{GaP}$ conduction band. Indeed, the resulting crystals were highly resistive at room temperature, with resistivity in the order of $10^{8}-10^{10} \Omega \mathrm{cm}$. The ingots came out as monocrystals with one exception, for the highest $\mathrm{Cr}$ content, when mono/poli structure was observed.

\section{Magnetic measurements}

Magnetization of the samples was measured as a function of magnetic field (up to $6 \mathrm{~T}$ ) and temperature $(2-100 \mathrm{~K}$ ) using a SQUID magnetometer. In general, all samples showed two dominant contributions, i.e., paramagnetic (PM) and diamagnetic ones. The magnetization was corrected for diamagnetic constant coming 
from GaP lattice. Its value was estimated from magnetization data performed on GaP reference sample. PM contribution proved to be well described by the Brillouin function with $S=l$. Most probably it originated from $\mathrm{Cr}$ incorporated to GaP lattice in $\mathrm{Cr}^{3+}\left(d^{3}\right)$ and $\mathrm{Cr}^{2+}\left(d^{4}\right)$ configurations with $S=1 / 2$ and $S=2$, respectively. In samples with the highest initial Cr-content $\left(6 \times 10^{20} \mathrm{~cm}^{-3}\right.$ in the melt, $4 \times 10^{17} \mathrm{~cm}^{-3}$ in the bulk) an antiferromagnetic (AFM) contribution to the total magnetization was also observed. It was found in magnetization measured as a function of magnetic field where at high temperatures $(T>100 \mathrm{~K})$ pronounced linear response was observed. Chromium, as it has been already mentioned, is known to be AFM element with $T_{\mathrm{N}}=312 \mathrm{~K}$ and its inclusions were most probably responsible for AFM contribution to magnetization. Presence of chromium AFM response in total magnetization was corroborated by susceptibility measurements.

Close inspection of the magnetization data obtained at low temperatures $(T<6 \mathrm{~K})$ revealed as well, unexpectedly, dominant, quite strong diamagnetic response at low magnetic field. In order to investigate deeper this phenomenon, magnetization of the samples was measured as a function of magnetic field at low temperatures (Fig. 1).

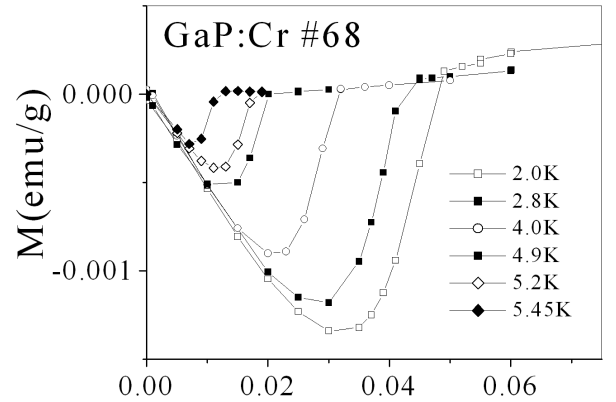

Fig. 1 $\mathrm{B}(\mathrm{T})$

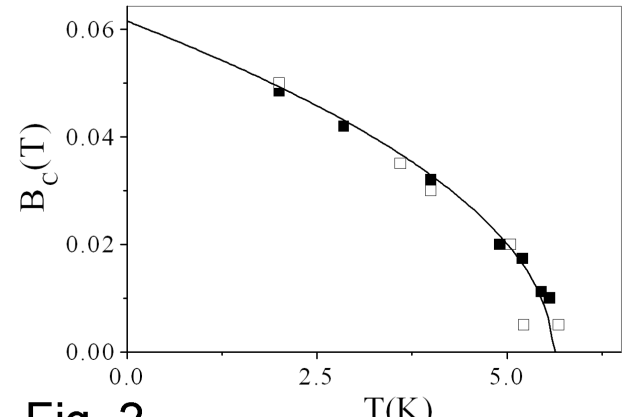

Fig. 2

Fig. 1. Magnetization of the sample from the ingot with the highest intentional $\mathrm{Cr}$ doping, plotted as a function of magnetic field for different temperatures.

Fig. 2. The critical field $B_{\mathrm{C}}$ dependence on temperature. The $B_{\mathrm{C}}$ was determined as a magnetic field at which increasing slope of magnetization curve (see Fig. 1) intersects with magnetic field axis.

We also observed diminishing of diamagnetic response at a particular critical magnetic field $B_{\mathrm{C}}$ as well as $B_{\mathrm{C}}$ dependence on temperature. These are fingerprints of superconductive phase of any material. The experimental magnetization curve did not allow for straightforward differentiation between type I and type II superconductor. We are rather inclined to type II superconductor but with mixed state in rather narrow range of magnetic field. The $B_{\mathrm{C}}$ dependence on temperature is shown in Fig. 2 . 


\section{X-ray measurements}

X-ray measurements on (111) oriented samples employed high resolution multicrystal diffractometer measurements as well as X-ray topography in classical transmission mode. Since the samples were of (111) orientation we employed (111) and (333) type reflections to collect data for rocking curve measurements and reciprocal space maps. The used multicrystal setup included Hart-Bartels-type four-crystal channel cut beam conditioner as a primary X-ray optics element and two-crystal analyzer placed in front of the diffracted beam detector. All measurements were made in so-called symmetric diffraction geometry with the $0.15405 \mathrm{~nm}$ wavelength used. For the transmission topography we employed classical Lang topography arrangement, also in so-called symmetrical geometry but in this case in transmission mode. In this case we used (220) reflection which diffracted from (110) type crystallographic planes perpendicular to the sample main surfaces. Diffractometric results, both rocking curves and reciprocal space maps, indicated good overall crystal quality of the samples with some possible small lattice parameter variation. Most fruitful result came out from transmission topography which revealed network of "cellular structure" (see Fig. 3) usually observed in LEC grown GaAs crystals. This type of structure is well known to contain within the cell walls segregated intentional or non-intentional dopants and other defects.

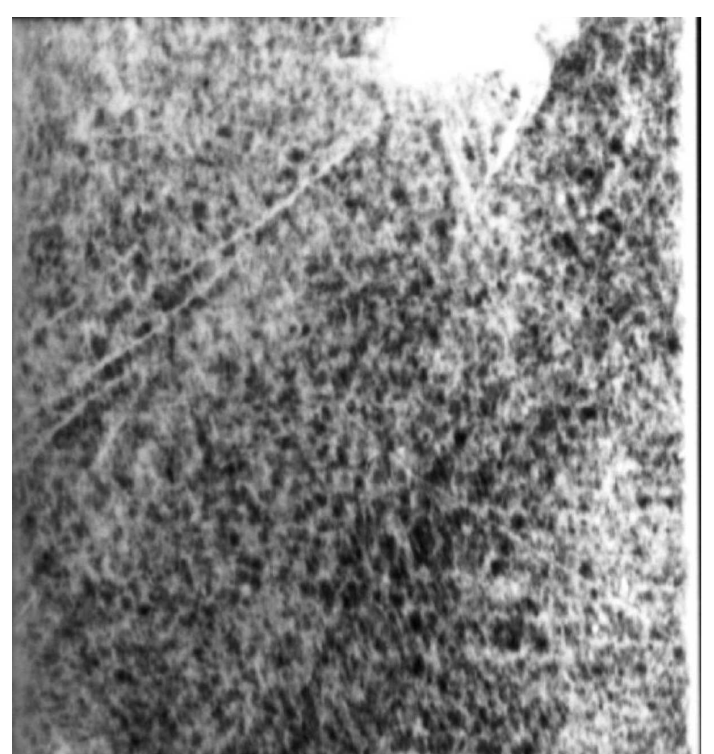

Fig. 3. X-ray transmission topography of highly Cr doped GaP, obtained for 220 reflection (0.070926 nm radiation). Complicated network of the "cellular structure" type image is observed. The image width is $8 \mathrm{~mm}$. 


\section{Conclusions}

The observed results of magnetic measurements, indicating superconductivity of GaP highly doped with Cr, most probably could be related to the presence of $\mathrm{Cr}$ in host GaP lattice. The cellular structure found for the studied samples could mean Cr micro-precipitates or Cr micro-platelets embedded in cell walls. There is scarce literature data about superconductivity of Cr. As it was mentioned in Introduction, superconductivity phenomenon has not been observed in bulk $\mathrm{Cr}$ while thin Cr-layers embedded in Au-layers were shown to be superconducting up to about $3 \mathrm{~K} \mathrm{[7]}$. This is a first report of $\mathrm{Cr}$ superconductivity in semiconductor host. It seems worth further studies because of interesting physics as well as possible applications including superconducting interconnections, junctions, and switches in integrated semiconductor-based devices.

\section{References}

[1] H. Kamerlingh Onnes, Akad. Van Wetenschappen 14, 113, 818 (1911).

[2] P. Dai, B.C. Chakoumakos, G.F. Sun, K.W. Wong, Y. Xin, D.F. Lu, Physica C 243, 211 (1995).

[3] C. Buzea, K. Robbie, Supercond. Sci. Technol. 18, R1 (2005).

[4] P.H. Schmidt, R.N. Castellano, H. Barz, B.T. Matthias, Phys. Lett. A 41, 367 (1972).

[5] M.B. Brodsky, A.J. Freeman, Phys. Rev Lett. 45, 133 (1980).

[6] M.B. Brodsky, J. Appl. Phys. 52, 1665 (1981).

[7] M.B. Brodsky, P. Marikar, R.J. Friddle, L. Singer, C.H. Sowers, Solid State Commun. 42, 675 (1982).

[8] X. Jian-hua, A.J. Freeman, T. Jalborg, M.B. Brodsky, Phys. Rev. B 29, 1250 (1984).

[9] L.R. Sill, M.B. Brodsky, S. Bowen, H.C. Hamaker, J. Appl. Phys. 57, 3663 (1985).

[10] A.M. Marsh, D.A. Williams, J. Vac. Sci. Technol. A, Vac. Surf. Films 14, 2577 (1996) 